\title{
A case of multiple immune toxicities from Ipilimumab and pembrolizumab treatment
}

\author{
Malik Asif Humayun, ${ }^{1}$ Ruth Poole ${ }^{2}$ \\ ${ }^{1}$ Royal Bournemouth Hospital, Bournemouth, ${ }^{2}$ Poole Hospital, NHS Foundation Trust, Poole; UK
}

\section{Dear Sir,}

Monoclonal antibodies have revolutionized the management of complex and challenging human diseases such as malignancies as well as haematological, rheumatologic and other autoimmune conditions over the last three decades. ${ }^{1}$ At the same time, they are associated with a significant degree of immune-mediated disorders, and endocrinopathies are no exception.

Ipilimumab is an immune modulation biological agent which acts on anti-cytotoxic T-lymphocyteassociated antigen 4 (CTLA-4) and is licensed for the treatment of metastatic melanomas. ${ }^{1}$ Pembrolizumab is a programmed death receptor (PD 1) blocking agent and is indicated for the treatment of patients with unresectable or metastatic melanoma and disease progression following ipilimumab and if BRAF gene mutation (the gene that makes a protein called B-Raf which is serine/threonine-specific protein kinase) is positive. ${ }^{2,3}$ Pituitary dysfunction is a well-recognised side effect of ipilimumab and is also very occasionally seen $(0.5 \%)$ with pembrolizumab. ${ }^{3,4}$ The incidence of hypophysitis induced by CTLA- 4 mAbs is reported to be $0-17 \% .^{1}$ The exact mechanism by which these therapies cause hypophysitis remains unknown but it seems to be immune in nature. ${ }^{1}$

\section{Address for correspondence:}

Dr. Malik Asif Humayun, MRCP, FCPS, Department of

Diabetes \& Endocrinology, Royal Bournemouth Hospital,

Castle Lanes East, Bournemouth, BH7 7DW, United Kingdom;

E-mail: doctormalikasif@gmail.com

Received: 22-12-2015, Accepted: 27-01-2016
Patients who develop hypophysitis usually present with subacute symptoms including headaches, visual symptoms, lethargy, weight gain and a general feeling of unwellness. There is no specific timescale after starting CTLA-4 mAb treatment at which patients will develop hypophysitis. ${ }^{5}$ There does not appear to be any specific hormone axis affected more than others. Diffuse pituitary enlargement on MRI is the most frequent radiological finding.

High-dose steroids and other hormone replacements improve outcomes and various reports have shown a favourable outcome in terms of clinical, biochemical and radiographic resolution of this condition without affecting the antitumour activity of these agents. ${ }^{6}$ The majority of clinicians have used intravenous steroids like dexamethasone but some centers have reported successful treatment with oral prednisone. ${ }^{7}$ Re-treatment with the same CTLA-4 mAb seems to be safe whenever indicated. ${ }^{1}$

Through this letter we report the case of a previously fit and healthy 55-year old man who was referred by his GP with an asymptomatic skin lesion on the lower back which had been growing in size for about six months. The rest of his past medical history was insignificant and he did not have any family history of diabetes or autoimmune conditions. The biopsy of the skin lesion confirmed the diagnosis of malignant melanoma which was BRAF positive. At the time of diagnosis he had metastatic disease in his inguinal lymph nodes and a staging CT scan revealed subcutaneous pelvic lymphadenopathy with possible 
low external iliac lymph node involvement. No metastatic disease was identified elsewhere; however, a PET scan, which was performed six months after his diagnosis, confirmed widespread metastases involving bone, lung, nodes and liver. At that time, he was treated with first-line palliative chemotherapy with dacarbazine. He achieved a favourable initial partial response to this treatment based on the repeat $\mathrm{CT}$ scan which confirmed healing of bone disease and reduction of size of the lung nodule and of that of the left inguinal lymph node group. The liver lesions were no longer visible.

A repeat CT scan after the completion of his firstline chemotherapy treatment confirmed some disease activity in the inguinal region, hence he was started on the second-line treatment with ipilimumab. However, after four cycles over two weeks of ipilimumab treatment he presented with fatigue and blurring of vision and was feeling generally unwell. A full pituitary profile at that point confirmed pan-hypopituitarism (Table 1). He was started on $4 \mathrm{mg}$ of dexamethasone with intention to treat his hypocortisolism and suppress the immune reaction in the pituitary. All his symptoms, including blurring of vision, improved rapidly and his dexamethasone dose was reduced to $2 \mathrm{mg}$ once daily after one week. His pituitary profile, which was repeated after four weeks, showed a normal pituitary function (Table 1). He did not have an MRI of his pituitary at the time of presentation; however, an MRI four weeks after his initial clinical presentation and treatment with dexamethasone showed a normal appearance of the pituitary. At that point, his

Table 1. Pituitary profile at the time of diagnosis and six weeks later

\begin{tabular}{lcccc}
\hline & $\begin{array}{c}\text { Hormone } \\
\text { profile at } \\
\text { the time of } \\
\text { diagnosis }\end{array}$ & $\begin{array}{c}\text { Hormone } \\
\text { profile } \\
\text { six weeks } \\
\text { later }\end{array}$ & Units & $\begin{array}{c}\text { Reference } \\
\text { range }\end{array}$ \\
\hline FT4 & 6 & 15 & $\mathrm{pmol} / \mathrm{L}$ & $(10-22)$ \\
TSH & 0.52 & 2.3 & $\mathrm{mU} / \mathrm{L}$ & $(0.3-5.50)$ \\
Testosterone & $<0.1$ & 13.3 & $\mathrm{nmol} / \mathrm{L}$ & $(7-26)$ \\
LH & 0.8 & 7 & $\mathrm{iU} / \mathrm{L}$ & $(1-10)$ \\
FSH & 4.6 & 10.6 & $\mathrm{iU} / \mathrm{L}$ & $(1-12)$ \\
Cortisol & $<18$ & On replacement & $\mathrm{nmol} / \mathrm{L}$ & $(200-700)$ \\
Prolactin & Not done & 181 & $\mathrm{mU} / \mathrm{L}$ & $(86-324)$ \\
IGF-1 & Not done & 212 & $\mathrm{ug} / \mathrm{L}$ & $(94-231)$ \\
\hline
\end{tabular}

dexamethasone was switched to hydrocortisone with a view to keeping him on this treatment a little longer, due to the risk of recurrence of his hypophysitis, and then gradually weaning him off his steroid treatment. A repeat CT scan six weeks after the completion of his ipilimumab treatment confirmed that his disease had progressed. He embarked on third-line treatment with pembrolizumab. His disease remained stable on the repeat $\mathrm{CT}$ imaging, but six months after starting pembrolizumab he developed increasing effusion of his knee joints. Clinically there was gross synovitis in both knees. His inflammatory markers were very high but anti-cyclic citrullinated peptide antibodies and other auto-antibody screens were negative. There was no evidence of infective arthritis. He was diagnosed with pembrolizumab-associated immune arthropathy and was accordingly treated with systemic and intra-articular steroids, which improved his symptoms significantly. His disease remained stable on a repeat CT scan which was performed after six cycles of his pembrolizumab treatment; therefore, it was decided to continue with this treatment.

After nine cycles of his pembrolizumab treatment he presented to the emergency department with symptoms of tiredness, polyuria and polydipsia. His serum glucose was $42 \mathrm{mmol} / \mathrm{l}$, a venous blood gas showed a $\mathrm{pH}$ of 6.93 and his capilliary ketone levels were $6.8 \mathrm{mmol} / \mathrm{l}$. He was treated according to the hospital diabetic ketoacidosis (DKA) protocol and made a good recovery. At that point his HBA1c was $93 \mathrm{mmol} / \mathrm{mol}$. He was diagnosed with type 1 diabetes and commenced on basal bolus insulin. GAD (glutamic acid decarboxylase) and islet cell antibody levels were undetectable. His glycaemic control improved significantly and remained stable after discharge.

He also reported having pain in his right hip and an X-ray of his hip showed some osteoarthritic changes but also significant thinning of the cortex. An MRI of the femur and pelvic area demonstrated a moderate effusion with surrounding soft tissue oedema and pre-existing degenerative change. There was no evidence of fracture or metastatic disease and imaging appearance was more in keeping with bone marrow inflammation and oedema likely secondary to osteitis due to immune toxicity of pembrolizumab treatment. He was given ten cycles of pembrolizumab treatment in total, after which it was discontinued in 
view of the evidence of disease progression and immune toxicities. He also developed a maculopapular rash approximately six weeks after his tenth cycle of pembrolizumab treatment, which was thought to be secondary to his treatment. The rash disappeared without any treatment after two weeks.

Type 1 diabetes mellitus (T1DM), including diabetic ketoacidosis, has been reported in $0.1 \%$ of patients across clinical studies with pembrolizumab in approximately 5000 patients. ${ }^{4}$ This risk has not been described in the drug literature of pembrolizumab. We have found only one previously published case report of T1DM due to pembrolizumab. ${ }^{2}$ The latter patient had positive anti-GAD and ICA, suggesting a possible association of PD-1 inhibitors with autoimmune diabetes. Interestingly, these antibodies were negative in our patient. Our patient presented with acute DKA as a first presentation of diabetes and this favours the possibility of an insulin-deficient state like type 1 diabetes as opposed to type 2 diabetes or latent autoimmune diabetes of adulthood, which has a more gradual onset. The previously cited case presented with T1DM after three doses of pembrolizumab, while our patient presented after the ninth infusion of pembrolizumab. Both these cases presented with DKA as a first manifestation of diabetes. There was no family history of T1DM in either of the cases. Of note, our patient's insulin requirement did not change after completion of his pembrolizumab treatment (over the three months follow-up period), hence we suspect that unlike several other immune toxicities related to mAbs treatment, diabetes does not seem to resolve spontaneously after termination of the treatment.

Our patient also developed other immune-mediated toxicities, including arthropathy and osteitis. Arthropathy is also a very rare side effect of PD-1 inhibitors and so far only two cases have been reported in the literature. ${ }^{8}$ It responded well to the intra-articular and systemic steroid therapy and the patient did not require any further treatment after the end of his pembrolizumab treatment.

It is also of interest that our patient developed several different immune-toxicities secondary to his mAbs treatment for malignant melanoma and all of them resolved spontaneously after cessation of treatment except for his diabetes. Our case also heightens our awareness of complications associated with the clinical use of these agents and may be considered as a prototypical model for future research into the understanding of these immune toxicities.

\section{CONCLUSION}

Although not very commonly encountered in the past, hypophysitis and other metabolic and endocrine disorders secondary to the use of monoclonal antibody treatment will be increasingly encountered by the acute medical team, oncologists and endocrinologists.

Hypophysitis and T1DM can develop due to immune toxicities of modern anticancer treatments and, if not promptly diagnosed and properly treated, may be life-threatening due to secondary adrenal insufficiency and DKA, respectively, the key being rapid diagnosis and therapeutic intervention. This can be achieved through a close level of communication between the relevant specialties and patient education.

Patients should be educated and may be provided with a safety card about the symptoms and the likely immune toxicities from their anticancer medication.

Clinicians, especially emergency and acute medical teams, should be vigilant about the clinical features of such potential life-threatening toxicities.

There is also a need to discover new predictive biomarkers of both activity and toxicity of these agents.

\section{REFERENCES}

1. Torino F, Barnabei A, Paragliola RM, Marchetti P, Salvatori R, Corsello SM, 2013 Endocrine side-effects of anti-cancer drugs: mAbs and pituitary dysfunction: clinical evidence and pathogenic hypotheses. Eur J Endocrinol 169: R153-164.

2. Martin-Liberal J, Furness A, Joshi K, Peggs KS, Quezada SA, Larkin J, 2015 Anti-programmed cell death-1 therapy and insulin-dependent diabetes: a case report. Cancer Immunol Immunother 64: 765-767.

3. Gangadhar TC, Salama AK, 2015 Clinical applications of PD-1-based therapy: a focus on pembrolizumab (MK-3475) in the management of melanoma and other tumor types. OncoTargets Ther 8: 929-937.

4. http://www.accessdata.fda.gov/drugsatfda_docs/label /2014/125514lbl.pdf

5. Weber JS, Dummer R, de Pril V, Lebbé C, Hodi FS, 2013 Patterns of onset and resolution of immune-related adverse events of special interest with ipilimumab: 
detailed safety analysis from a phase 3 trial in patients with advanced melanoma. Cancer 119: 1675-1682.

6. Dillard T, Yedinak CG, Alumkal J, Fleseriu M, 2010 Anti-CTLA-4 antibody therapy associated autoimmune hypophysitis: serious immune related adverse events across a spectrum of cancer subtypes. Pituitary 13:29-38.

7. Rodrigues BT, Otty Z, Sangla K, Shenoy VV, 2014
Ipilimumab-induced autoimmune hypophysitis: a differential for sellar mass lesions. Endocrinol Diabetes Metab Case Rep 2014: 140098.

8. Chan MM, Kefford RF, Carlino M, Clements A, Manolios N, 2015 Arthritis and tenosynovitis associated with the anti-PD1 antibody pembrolizumab in metastatic melanoma. J Immunother 38: 37-39. 